\title{
Significados y efectos en torno a la evaluación de los aprendizajes escolares*
}

\author{
Meanings and Effects around the Assessment \\ of School Learning
}

\author{
Horacio Ferreyra ${ }^{* *}$, Fabiana Castagno ${ }^{* * *}$, María Alejandra Salgueiro ${ }^{* * * *}$ \\ y Susana del Milagro Tucci* ${ }^{* * * *}$
}

Fecha de recepción: 16 de abril de 2012

Fecha de revisión: 24 de abril de 2012

Fecha de aprobación: 28 de mayo de 2012

* $\quad$ Artículo de reflexión correspondiente al trabajo investigativo del Grupo de Práctica Evaluativa de la Universidad de Córdoba, Argentina.

** Doctor en Educación y profesor e investigador de la Universidad Católica Argentina. Posdoctorado en Ciencias Sociales por la Universidad Nacional de Córdoba y posdoctorado en Ciencias Sociales y Educación por la Universidad Autónoma Metropolitana, México, profesor e investigador de la Universidad Santo Tomás, Colombia. Subsecretario de Promoción de Igualdad y Calidad Educativa del Ministerio de Educación de la provincia de Córdoba, Argentina. Correo electrónico: hferreyra@coopmorteros.com.ar

*** Licenciada en Ciencias de la Educación, Universidad Nacional de Córdoba, Argentina. Integrante del Área de Evaluación de la Calidad Institucional, subsecretaría de Promoción de Igualdad y Calidad Educativa, Ministerio de Educación de la Provincia de Córdoba. Profesora e investigadora de la Universidad Nacional de Córdoba, Argentina. Correo electrónico: fabianacastagno@hotmail.com

**** Magíster en Administración Educacional, Universidad de Playa Ancha, Chile. Integrante del Área de Formadores de Directivos de Institutos de Formación Docente, Instituto Nacional de Formación Docente, Ministerio de Educación Nacional e integrante del Área de Evaluación de la Calidad Institucional de la Subsecretaría de Promoción de Igualdad y Calidad Educativa, Ministerio de Educación de Córdoba. Docente de institutos de formación de docentes de la Provincia de Córdoba. Correo electrónico: asalguei@live.com.ar

***** Licenciada y profesora en Pedagogía y Psicopedagogía, Universidad Nacional de Córdoba, Argentina. Es referente del Área de Evaluación de la Calidad Institucional de la Subsecretaría de Promoción de Igualdad y Calidad Educativa, Ministerio de Educación de la Provincia de Córdoba, docente de la Universidad Nacional de Córdoba y de la Universidad Nacional de Chilecito, Argentina. Correo electrónico: susanatucci26@hotmail.com 


\section{Resumen}

El presente artículo tiene por objetivo aportar algunas reflexiones en torno a las prácticas de evaluación y los sentidos y efectos que estas producen. Plantea una puesta en relación con las trayectorias escolares y las acciones e intervenciones docentes. Se pone en relieve la dimensión ético política, la responsabilidad, la confianza y la autoridad que en toda práctica evaluativa se juega, generando condiciones de posibilidad, de crecimiento, de potencialidad o no en los estudiantes evaluados.

Asimismo, sobre la base de estos planteos se expone un trabajo llevado a cabo para revisar y fortalecer los procesos de autoevaluación institucional en las escuelas de la Provincia de Córdoba, Argentina.

Palabras clave: Evaluación de aprendizajes, trayectorias escolares, enseñanza, significados.

\section{Abstract}

The aim of the present paper is to contribute with some observations on assessment practices and the effects they produce. It establishes a relationship with the school experience, the teachers' actions and teaching interventions. The ethical-political realm, the responsibility, the trust and the authority are highlighted because they might generate the conditions of possibility, growth and potentiality in the assessed students.

Also, on the basis of these suggestions, a field work carried out to review and strengthen the processes of institutional auto-evaluation in the schools of the Province of Córdoba - Argentina is presented.

Keywords: Learning assessment, school experience, teaching, meaning 


\section{Ensayando otras maneras de pensar la evaluación}

Si hay algo que quienes trabajamos en educación no podemos soslayar es la discusión acerca de la evaluación de los aprendizajes de los estudiantes, discusión que generalmente ronda alrededor de los instrumentos, los criterios y los momentos.

Mucha bibliografía nos plantea la importancia de discutir y acordar criterios de evaluación, de cómo elaborar buenos instrumentos, de cuáles son los momentos y los tipos de evaluación que toda educación formal requiere.

Sin ánimo de cuestionar estos aportes que han tenido cierta relevancia para iluminar algo sobre la evaluación escolar, lo que nos interesa enfatizar en este artículo es la dimensión ética y política que toda acción evaluativa conlleva y que sostiene las cuestiones de orden instrumental.

Porque consideramos que el solo construir buenos instrumentos, el acuerdo sobre criterios de evaluación y la instalación de diferentes tipos de evaluación no agota el tratamiento de esta cuestión. O mejor dicho, toda acción educativa, y aquí ubicamos sin duda la evaluación, así sea el armado de una simple prueba escrita, es una acción ética y política ya que produce efectos.

Pensar la evaluación desde esta perspectiva o mirada nos ayudaría a visibilizar sentidos, significados, posiciones, ideologías, convicciones que no siempre se discuten cuando tenemos que evaluar a nuestros alumnos. Las preguntas acerca de para qué y por qué evaluamos y qué efectos producen las evaluaciones en las trayectorias de los alumnos, nos exigen poner en claro principios, valores y sus respuestas no pueden reducirse a una definición de manual, requiere volver a pensar la educación, volver a pensar los procesos de aprendizaje y qué los sostienen.

Si bien esta discusión se viene dando en los últimos años, nos parece que podríamos acercar algunas ideas que intentan acompañar este análisis sin pretensión de agotarlo.

\section{La evaluación en el marco de una acción educativa. Autoridad - experiencia - confianza}

Una primera cuestión, que hoy al menos en los discursos está instalada, es que la evaluación es parte del proceso educativo, es "una instancia más de aprendizaje". Las preguntas que se nos presentan son: ¿esto es posible?, ¿se viene dando así, siempre, en toda evaluación?, ¿se aprende algo en el momento de ser evaluado?

Se nos ocurre ligar la idea de evaluación aprendizaje con el concepto de autoridad.

La concepción clásica de autoridad alude a lo que hace crecer, lo que permite que algo o alguien aumente o crezca. En esta línea podríamos agregar la autoridad como habilitación de la palabra de uno y otro, como posibilidad de sostener lo viejo y al mismo tiempo dar lugar a lo nuevo, un crecimiento.

La pregunta que se nos ocurre ahora es ¿la evaluación es una instancia de autorización de 
la palabra de quien es evaluado, es una oportunidad de crecimiento?, ¿es una invitación a revisar, pensar, producir, entramar, relacionar y de seguir caminando?, ¿o por el contrario es lo que paraliza, inmoviliza, acalla, reitera, repite sin lugar a lo nuevo?, ¿lo que califica (o descalifica) a alguien determinando un destino inexorable, una trayectoria escolar "accidentada", interrumpida, deshecha?

Algunos autores, como Beatriz Greco, asocian la autoridad con el reconocimiento y la legitimidad, lo cual lleva a "aceptar aquello que la autoridad dispone o determina sin oponerse, teniendo la posibilidad de reaccionar en su contra" (2007, p. 40).

La evaluación en su faceta formal cuenta con el reconocimiento como para imponerse, muy pocos cuestionan su legitimidad (salvo en situaciones extremas en las que un alumno o un padre "reacciona en su contra" cuando los resultados no son favorables). Desde el punto de vista social y didáctico se reconoce la importancia de dar cuenta de los procesos formativos que los estudiantes están desplegando, así como de los resultados alcanzados. Quizá lo que podríamos seguir pensando es si solo nos importa constatar procesos y resultados o si además puede generar otros efectos: la movilización de pensamientos, la reconstrucción e integración de conocimientos, el análisis de los propios procesos de aprendizaje. En definitiva, la preocupación es si una evaluación tiene potencial formativo.

Cuando decimos esto, una evaluación que forma, educa, acompaña y sostiene la formación o educación de un sujeto, nos referimos a una evaluación que da experiencia. Jorge Larrosa desarrolla con claridad la idea de experiencia y la asocia fuertemente con la de educación: no hay educación sin experiencia. Para él la experiencia es eso que nos pasa, que nos deja marca, huella, que provoca una transformación, un cambio en cada uno (2009, pp. 14-15).

Podríamos pensar entonces si las evaluaciones dan experiencia a los estudiantes, si habilitan, autorizan, provocan un acontecimiento del orden del pensamiento, de la acción que hace crecer, que hace aumentar capacidades, posibilidades, aprendizajes.

Walter Kohan pone atención a las preguntas que hacemos los docentes y plantea si las preguntas que hacemos realmente interrogan al otro, "preguntamos para escuchar una única respuesta que nos conforma, que ya sabíamos antes de lanzar la pregunta" (2004, p. 77).

No estaría mal analizar las preguntas que hacemos al momento de evaluar o las consignas que proponemos en una tarea, trabajo, actividad evaluativa: ¿son preguntas/consignas que interrogan, que provocan experiencia, que ofrecen la oportunidad de construir algo nuevo, de pensar y pensarse distinto, o son preguntas que pretenden reforzar, constatar lo que ya sabemos del alumno, de su capacidad, de su posibilidad?

La necesidad de solo constatar o confirmar un resultado nos estaría alejando de la confianza que se requiere para sostener una relación pedagógica, se instala por el contrario la sospecha de que el otro no va a poder más que lo que ya sabemos que puede. 
Restablecer la confianza en una evaluación probablemente coloque al estudiante en un lugar de potencia, porque el docente apuesta a su saber, a su capacidad, a su condición de persona que se está formando, que está aprendiendo. Lo expresamos en gerundio porque el aprendizaje es un acontecer que se va desplegando continuamente y que no termina, no culmina con una evaluación, en un momento establecido y arbitrario.

Nos hemos acostumbrado a disociar, a fragmentar, desarticular conceptos, acciones, espacios. A veces, la evaluación ha corrido esta suerte, desacoplándose de los procesos de enseñanza y del aprendizaje. Volverla a constituir como parte del aprendizaje es otra vez un desafío.

\section{La evaluación como analizador de las prácticas de enseñanza. Responsabilidad}

Sabemos que cualquier fenómeno evaluativo arroja ciertos datos acerca de lo que logró o no logró un alumno en términos de aprendizaje, aunque podríamos relativizar esta constatación. Porque no siempre los resultados de una prueba escrita, lo que expresa o puede decir un alumno en una "lección oral" dan cuenta cabalmente de su aprendizaje.

Lo que sí queremos afirmar es que resulta un analizador muy potente de la enseñanza; no solo en su versión técnica sino como venimos diciendo, de los propósitos e intencionalidades que un docente, una institución escolar y un sistema educativo pretende impulsar.
La evaluación es el material que tenemos a mano para "descomponer sus elementos", para encontrar los "sentidos y significados ocultos" como lo plantea Georges Lapassade (1979, pp. 17-18). Lo que queremos decir es que los modos de evaluar hablan, muestran, develan los posicionamientos políticos y éticos de los docentes, sus marcos teóricos, sus ideologías, sus maneras de hacer y concebir la educación.

Y cuando hablamos de modos de evaluar nos referimos a qué se evalúa, cómo, cuándo, para qué, por qué, a quién y, sobre todo, la lectura que se hace de las respuestas de los alumnos y qué se hace con esa lectura: constatar hipótesis sobre el rendimiento de los alumnos, colocar una calificación muchas veces arbitraria, sellar la promoción de alguien, determinar un destino.

Leímos y decimos que la evaluación no solo es del estudiante y del aprendizaje, sino también del docente y de la enseñanza; sin embargo, en los hechos pareciera que los bajos rendimientos de los estudiantes poco tienen que ver con la acción de un docente o de una institución escolar, seguimos con la lógica de culpabilizar a los alumnos de los malos resultados.

Este corrimiento nos lleva a plantear la idea de responsabilidad. En este punto volvemos a recuperar el lugar de la evaluación en el sentido que lo venimos proponiendo: como posibilidad de pensamiento, de aprendizaje de nuestras propias prácticas pedagógicas. Se trata entonces de una responsabilidad que llame al pensamiento, a la reflexión, a la acción para darnos nosotros también la oportunidad de crecer y desarrollarnos profesionalmente. 


\section{Una propuesta desarrollada en la provincia de Córdoba, Argentina}

En el año 2009 la provincia de Córdoba llevó a cabo una serie de acciones de fortalecimiento de los procesos de evaluación en las escuelas, priorizándose la mirada sobre las trayectorias escolares, pues su abordaje resultaba insoslayable a la hora de pensar las políticas educativas y las intervenciones pedagógicas. Al respecto, se fueron planteando algunas preguntas que permitieron conceptualizar las trayectorias como una cuestión institucional, no individual en la línea que lo hacen Sandra Nicastro y Beatriz Greco (2009, pp. 2324) y que se fueron formulando de la siguiente manera: ¿cómo son las trayectorias reales de los estudiantes?, ¿cuáles son los puntos críticos en esos recorridos?, ¿qué información expresantraducen estas trayectorias?, ¿qué evidencias nos permiten describir, interpretar y reflexionar sobre las trayectorias de los estudiantes? Sin lugar a dudas, estos interrogantes abrieron el análisis de aquellos aspectos institucionales (tiempos, espacios, proyectos formativos, prácticas pedagógicas, modos de vinculación) que configuran itinerarios determinados para los estudiantes. Entonces, se vuelve a preguntar: ¿qué prácticas de la institución sostienen, acompañan las trayectorias de los estudiantes y cuáles no?, ¿qué aspectos dan cuenta de las condiciones y prácticas institucionales que acompañan, sostienen o no sus trayectorias?

Mirar las trayectorias sin duda exige poner en relación los resultados y procesos de aprendizaje alcanzados por los estudiantes con los proyectos y propuestas pedagógicas, con las maneras de evaluar a los estudiantes y evaluar lo que cada docente enseña y cómo lo hace. Es decir, existen mediaciones que condicionan determinados recorridos. Por tanto, la lectura de las trayectorias, al igual que el análisis de los resultados, exige -a su vez- analizar las prácticas educativas que promueven las escuelas, sus dispositivos pedagógicos (programas, planificaciones, actividades, materiales, recursos), sus modos de evaluar. Dicho de otro modo, implica poner en tensión las trayectorias, en términos de procesos y resultados escolares con las variables de orden pedagógico, es decir, con la enseñanza como tarea de cada docente y como asunto institucional.

Se trabajó con todos los supervisores de distintos niveles y modalidades de la provincia de Córdoba por ser considerados clave en el acompañamiento y orientación de las escuelas a su cargo para mirarse, interpelarse y revisar sus prácticas. Esta decisión fue una apuesta para fortalecer esa dimensión del trabajo supervisivo en las instituciones escolares. Buscó también generar nueva información y volver a leer datos que producen en el marco de sus rutinas y reponer su lugar como recursos con un alto valor potencial para repensarse.

Para ello se elaboraron tres documentos con el propósito de abrir o profundizar el análisis del accionar institucional a fin de recuperar, revisar el sentido de las prácticas de evaluación, para ser más precisos, de las prácticas de autoevaluación a la luz de los planteos efectuados en los apartados precedentes: la dimensión ético - política, la de la experiencia, la confianza, la autoridad y la responsabilidad. 
Dichos documentos estimularon la reflexión y el debate sobre estos aspectos y fueron acompañados de una serie de categorías e indicadores que dieran cuenta de la situación escolar de los estudiantes a partir de la utilización de la línea de base como herramienta, no solo abordada desde su costado instrumental sino en su potencial epistémico, esto es, para generar explicaciones y producir pensamientos.

El primero hizo foco en las trayectorias escolares de los estudiantes, en tanto construcción acerca de la cual la escuela tiene para decir, analizar, pensar y operar. A través de este concepto se pretendió instalar un conjunto de interrogantes con respecto a los procesos y resultados de aprendizaje y el lugar que la escuela asume ante ellas, en orden a potenciar las capacidades de los estudiantes, en los sentidos que para ellos adquiere el "estar ahí", el "habitarla".

El segundo enfatizó las decisiones, acciones e intervenciones que la escuela a través de sus docentes, propuestas y proyectos pedagógicos lleva a cabo y generan condiciones para la construcción de las trayectorias de los alumnos.

El tercero abordó las relaciones e intercambios que cada escuela establece con el "afuera", con la(s) familia(s), la diversidad cultural, como también el clima que se respira y que habilita o no la formación y el aprendizaje en las instituciones educativas.

Con el propósito de analizar las trayectorias y su evaluación se formularon algunos interrogantes:
¿Qué trayectorias escolares posibilita/promueve la institución?, ¿qué trayectorias posibilitan las maneras de evaluar los procesos y resultados educativos de los estudiantes?, ¿qué efectos producen los modos de evaluar en el rendimiento de los estudiantes?, ¿de qué manera incide el pasado escolar de cada estudiante en las expectativas de los docentes y de la institución sobre sus aprendizajes?, ¿cuál es el potencial formativo de las propuestas de evaluación?

¿Qué tipos de evaluación acompañan el aprendizaje de los estudiantes y lo ayudan a crecer?, ¿qué tipo de marcas o expresiones lingüísticas de la evaluación aparecen en las producciones escritas, ayudan a lograr mejores comprensiones, a potenciar sus aprendizajes?

¿Qué relaciones, "puentes", articulaciones se dan entre las instancias evaluativas y los procesos de aprendizaje?, ¿cómo se mira al estudiante que tiene un rendimiento bajo?, ¿de qué manera se interviene cuando los resultados educativos no son los deseables?

\section{Conclusión}

A lo largo del artículo compartimos una mirada de la evaluación en general y de la evaluación de los aprendizajes, en particular, que hizo foco en los sentidos y efectos que estas prácticas producen en los estudiantes. Se puso de relieve la dimensión ética y política que los docentes ejercemos en el momento de valorar los procesos y resultados de aprendizaje, también, cuánto del orden del confiar, del ayudar a crecer, del desplegar y potenciar las capacidades 
de los estudiantes se concreta, se convoca en las instancias de evaluación.

Expusimos en consonancia con esas dimensiones un trabajo llevado a cabo durante 2009 que pretendió recuperar y potenciar las prácticas de evaluación en las escuelas de la provincia de Córdoba con el acompañamiento de los supervisores, con la firme convicción de que todo sistema educativo debe asumir la responsabilidad por las trayectorias escolares que se configuran en cada lugar, las que tienen bastante que ver con las maneras en que las escuelas evalúan a los estudiantes.

\section{Referencias}

Greco, B. (2007). La autoridad (pedagógica) en cuestión. Buenos Aires: Paidós.

Kohan, W. (2009). Infancia, política y pensamiento. Buenos Aires: Del Estante.

Larrosa, J. y Skliar, C. (2009). Experiencia $y$ alteridad en educación. Buenos Aires: Homo Sapiens.
Lapassade, G. (1979). El analizador y el analista. Barcelona: Gedisa.

Ministerio de Educación de la Provincia de Córdoba. Subsecretaría de Igualdad y Calidad Educativa. (2009a). La línea de base como herramienta en los procesos de autoevaIuación. Córdoba, Argentina.

Ministerio de Educación de la Provincia de Córdoba. Subsecretaría de Igualdad y Calidad Educativa. (2009b). La línea de base. Trayectorias escolares, acciones e intervenciones docentes. Córdoba, Argentina.

Ministerio de Educación de la Provincia de Córdoba. Subsecretaría de Igualdad y Calidad Educativa. (2009c). La línea de base: cultura - comunidad - profesionalización: algunas condiciones para la acción. Córdoba, Argentina.

Nicastro, S. y Greco, B. (2009). Entre trayectorias. Buenos Aires: Homo Sapiens. 